\title{
A utilização de softwares de análise de dados qualitativos: um mapeamento de teses em Educação Matemática
}

The use of qualitative data analysis software: a mapping thesis in mathematics education

\author{
Priscila Gleden Novaes da Silva ${ }^{1}$ \\ Rosangela Ramon ${ }^{2}$ \\ Alcione Cappelin ${ }^{3}$ \\ Sandra Maria Tieppo ${ }^{4}$ \\ Fernanda Marchiori Grave \\ Carbone Bruno Schmidt Krug ${ }^{6}$
}

\section{Resumo}

Este artigo caracteriza a utilização de softwares de análise de dados qualitativos em teses voltadas à Educação Matemática, desenvolvidas no Brasil, no período de 2010 a 2019, com o intuito de fundamentar as escolhas destes softwares a partir de caminhos já trilhados por outros pesquisadores. Para isso, foi realizado um mapeamento para desvelar dados identificadores das teses, bem como aspectos relacionados à adoção destes softwares. Foram analisadas 651 teses disponíveis na Biblioteca Digital Brasileira de Teses e Dissertações, das quais 31 compõem o corpus desta investigação, o que evidencia a pouca adoção destes softwares na área analisada. No corpus, foi observada a predominância da abordagem qualitativa e a identificação de nove softwares distintos, sendo os três mais utilizados: Atlas.ti, NVivo e CHIC, nesta ordem. A adoção dos softwares está atrelada ao potencial em relação à categorização, organização, visualização, manuseio dos dados, otimização de tempo e facilitação da análise, além de possibilitar exploração de grande volume e diversidade de dados.

Palavras chave: Educação Matemática; Pesquisa qualitativa; CAQDAS.

\section{Abstract}

This paper characterizes the use of qualitative data analysis software in doctoral theses about Mathematics Education developed in Brazil, from 2010 to 2019, to support the choices of these software from paths already followed by other researchers. For this, a mapping was made to identify the theses and the aspects related to the use of these software in the field.

\footnotetext{
${ }^{1}$ Universidade Federal Integração Latino-Americana | priscila.silva@unila.edu.br

${ }^{2}$ Instituto Federal de Santa Catarina | rosangela.ramon@ifsc.edu.br

${ }^{3}$ Centro Universitário de Pato Brancol alcione.cappelin@unidep.edu.br

${ }^{4}$ Universidade Federal do Paraná | sandra.tieppo@ufpr.br

5 Instituto Federal do Paraná | fermgrave@gmail.com

6 Instituto Federal do Paraná | prof13carbone@gmail.com
} 
The investigation retrieved 651 doctoral theses available in the Brazilian Digital Library of Thesis and Dissertations in which only 31 of them compose the corpus of this paper. This number evidences the little use of these software in the analyzed area. In the corpus, it was observed the predominance of the qualitative approach and the identification of nine different software, being three the most used: Atlas.ti, NVivo and CHIC, in that order. The adoption of software, as described by the authors, is linked to their potential regarding categorization, organization, visualization, data handling, time optimization and analysis facilitation and the possibility of enabling exploration of large volumes and diversity of data.

Keywords: Mathematics Education; Qualitative research; CAQDAS.

\section{Introdução}

Esta pesquisa é fruto de discussões realizadas no primeiro semestre de 2020, em disciplinas e atividades promovidas pelo Programa de Pós-Graduação em Educação em Ciências e Educação Matemática, da Universidade Estadual do Oeste do Paraná - Unioeste, com intuito de contribuir para a formação do pesquisador. As discussões instigaram aos doutorandos, autores deste artigo, inquietações acerca das próprias pesquisas, dentre elas a que diz respeito à adoção ou não de softwares de análise de dados qualitativos na etapa do tratamento dos dados, pois, como pesquisadores da área de Educação Matemática, esta é uma possível escolha a se levar em consideração durante uma pesquisa.

Boaler, Ball e Even (2003) atestam a importância de a formação de pesquisadores iniciantes ser nutrida por investigações de prática de pesquisas já realizadas, de modo que se perguntem a respeito do que está envolvido em uma investigação científica. O que os pesquisadores experientes mobilizam, consideram, experimentam e realizam em suas pesquisas? O foco, nessas práticas de pesquisa, ofereceria um caminho promissor para a preparação de pesquisadores iniciantes.

Nessa perspectiva, com o intuito de aprender com aqueles que já realizaram suas pesquisas, buscando identificar escolhas teóricas e metodológicas em nossa área, destacamos a importância e a relevância de serem desenvolvidas investigações que possam delinear panoramas sobre o conhecimento acumulado nessas produções, tendo em vista elucidar práticas e caminhos percorridos.

É nesse rumo que conduzimos nossa pesquisa, tendo como objetivo caracterizar a utilização de softwares de análise de dados qualitativos em teses voltadas à Educação Matemática, que foram desenvolvidas no contexto nacional, no período de 2010 a 2019. Para isso, propomos um mapeamento que busca desvelar dados identificadores das teses, bem como aspectos relacionados à adoção de software de análise de dados qualitativos.

Os softwares de análise qualitativa são designados pela expressão Computer Assisted Qualitative Data Analysis Software (CAQDAS) De acordo com Figueiredo e Maltempi (2019), os CAQDAS possibilitam melhorias quanto aos quesitos armazenamento, representação e integração, ou seja, novas perspectivas de visualização, análise e obtenção de dados. Além disso, o computador associado aos softwares proporciona transformação na atividade humana, alterando também o modo de se fazer pesquisa.

Existe uma diversidade de softwares de análise de dados qualitativos, o que, segundo Salvador et al. (2018), pode ser um dificultador para o pesquisador que precisa optar por um deles, tornando necessária uma análise criteriosa dos softwares disponíveis, de modo a selecionar um que se adeque ao seu objetivo e metodologia de pesquisa. 
Vislumbramos, desse modo, um contributo à nossa formação, como doutorandos em Educação Matemática e aos futuros pesquisadores dessa área ou correlata, no sentido de fundamentar suas escolhas com relação ao uso destes softwares. Ademais, com relação à pesquisa na área de Educação Matemática, esperamos lançar luz ao movimento em torno da utilização de softwares para análise qualitativa de dados, tendo em vista que não encontramos trabalhos com esse viés.

Em uma busca por estudos correlatos, encontramos o artigo de Salvador et al. (2019), no qual os autores realizaram uma análise em resumos de dissertações e teses na área da enfermagem entre 2013 a 2016, buscando verificar os softwares de análise de dados qualitativos utilizados. Além deste, o trabalho de Paula, Viali e Guimarães (2016) fez um levantamento em teses e dissertações de 2004 a 2015, buscando analisar o uso de CAQDAS nas pesquisas qualitativas de forma geral, com foco na análise textual.

Dessa forma, inicialmente justificamos a relevância do trabalho para a área da Educação Matemática, posteriormente, trataremos mais especificamente da pesquisa qualitativa e como os softwares podem auxiliar o pesquisador nesta etapa; em seguida, exibimos os passos metodológicos que realizamos para buscar as publicações e, por fim, exibimos os resultados e considerações sobre os trabalhos encontrados e analisados.

\section{Educação Matemática e Pesquisa Qualitativa}

A Educação Matemática é um campo de pesquisa jovem que abarca múltiplas relações e determinações entre ensino, aprendizagem e conhecimento matemático, configurando-se como campo profissional, sendo área de pesquisa teórica e, ao mesmo tempo, uma área de atuação prática (FIORENTINI; LORENZATO, 2006).

De acordo com Bicudo (2012) "a pesquisa em Educação Matemática mostra-se, na historicidade dessa área de inquérito, como efetuada, na grande maioria das vezes, de modo qualitativo" (p. 24). Garnica (2004) caracteriza pesquisa qualitativa como aquela que tem as características abaixo:

(a) a transitoriedade de seus resultados; (b) a impossibilidade de uma
hipótese a priori, cujo objetivo da pesquisa será comprovar ou refutar; (c)
a não neutralidade do pesquisador que, no processo interpretativo, vale-
se de suas perspectivas e filtros vivenciais prévios dos quais não consegue
se desvencilhar; (d) que a constituição de suas compreensões dá-se não
como resultado, mas numa trajetória em que essas mesmas
compreensões e também os meios de obtê-las podem ser
(re)configuradas; e (e) a impossibilidade de estabelecer regulamentações,
em procedimentos sistemáticos, prévios, estáticos e generalistas.
(GARNICA, 2004, p. 86)

Todavia, é de conhecimento, enquanto pesquisadores da área da Educação Matemática, que não há um consenso a respeito da forma de fazer pesquisa. Assim, compartilhamos do pensamento de Araújo e Borba (2004), os quais afirmam não existir uma regra única para a realização de pesquisas na nossa área, e que as características supramencionadas não devem ser vistas como regras, visto que, de forma recursiva, o próprio entendimento do que é pesquisa qualitativa está em movimento.

No que diz respeito à pesquisa qualitativa, Justo e Camargo (2014) salientam que os dados qualitativos são essencialmente significativos e diversificados, incluindo qualquer 
forma de comunicação humana escrita, auditiva ou visual. Pode-se trabalhar a partir de imagens, vídeos, áudios ou produções textuais, entre outras, sejam elas naturalmente existentes, ou produzidas pelo pesquisador, o que acaba gerando um grande volume de dados a serem analisados. No entanto, os autores afirmam que apesar da pluralidade de possibilidades disponíveis a esse tipo de pesquisa, considera-se que os materiais textuais têm sido o tipo de dado qualitativo mais comumente utilizado.

De acordo com Flick (2009), mesmo observando-se diferenças nas diversas abordagens possíveis, a análise e interpretação dos dados seria a essência da pesquisa qualitativa. Neste sentido, Figueiredo e Maltempi (2019) afirmam que "[...] a análise que o pesquisador apresenta pode ser entendida como uma visita guiada pelo labirinto dos dados, uma seleção daquilo que merece destaque em meio a um montante - até possivelmente extenso - de informações" (FIGUEIREDO; MALTEMPI, 2019, p. 197).

Assim, é importante destacar que, na maioria das vezes, o pesquisador se vê diante de um grande volume e tipos de dados, momento em que deve dispor de "[...] tempo e dedicação para conhecer tudo aquilo e escolher o que merece atenção [...]" (FIGUEIREDO; MALTEMPI, 2019, p. 197). É exatamente com vistas ao aprimoramento desta etapa da pesquisa, que vem ocorrendo a adoção de softwares de análise de dados qualitativos, no entanto, os mesmos autores afirmam que "[...] na área da Educação Matemática, cuja metodologia qualitativa é bastante empregada, nossa experiência nos mostra que a utilização de tais softwares é insignificante, apesar das vantagens evidentes" (2019, p. 198). Na sequência apresentamos uma discussão sobre aspectos relativos à adoção de softwares de análise de dados qualitativos.

\section{Software de análise de dados qualitativos}

Com o objetivo de auxiliar e facilitar a análise de dados qualitativos, cada vez mais são desenvolvidos CAQDAS, que podem ser classificados como softwares proprietários, ou seja, softwares os quais se faz necessário adquirir uma licença ou permissão de uso; softwares gratuitos, disponíveis livremente na rede de internet; e softwares livres, que também são gratuitos. Além disso, podem ser modificados e redistribuídos, conforme destacado por Schlosser, Frasson e Cantorani (2019).

Teixeira e Becker (2001) definem a utilização desses softwares como uma inovação, pela possibilidade de testar e relacionar hipóteses utilizando-se das mais variadas técnicas qualitativas e qualquer fonte de dados que possa ser convertido para texto. Esses softwares de análise de dados qualitativos, ao serem incorporados ao processo de pesquisa, possibilitam maior dedicação e exploração dos dados, pois viabilizam economia de tempo, bem como possibilitam a construção conceitual e teórica dos dados, por meio da estrutura formal que é gerada.

Quanto a sua utilização, é importante salientar que, não há uma linearidade entre o uso, sendo discutidas vantagens e desvantagens entre os pesquisadores da área. Uma das vantagens do uso de CAQDAS é a facilidade ao trabalhar com um grande volume de dados, como destacado por Lage (2011, p. 200): "[...] estas ferramentas computacionais tendem a ser especialmente úteis, quando se tem uma pesquisa qualitativa com grande volume de dados ou quando é necessário cruzar informações a partir dos atributos dos sujeitos de pesquisa". 
Moreira (2007, p. 58) apresenta como características padrões dos CAQDAS "[...] capacidade de codificação e recuperação de dados, capacidade de buscas automáticas", destacando, ainda, que codificar não é analisar, a análise deve ser feita pelo pesquisador. Justo e Camargo (2014, p. 43) apontam como vantagens o fato de que "[...] os computadores poderiam auxiliar no fornecimento de análises mais estáveis, objetivas e fidedignas, com menor interferência da subjetividade ou viés do pesquisador." No entanto, o uso do software pelo pesquisador não dispensa a análise e atenção aos dados.

Como desvantagens para o uso desses softwares tem-se, principalmente, o tempo que o pesquisador leva para aprender a usar o programa e se familiarizar com ele. Nesse sentido, Figueiredo e Maltempi (2019) ressaltam uma preocupação que o designer de ferramentas deve ter ao elaborar as interfaces desses programas, pois elas devem ser "[...] fluidas o suficiente para conciliar essa multiplicidade de esquemas de navegação e hierarquização e, ainda assim, manter uma experiência produtiva na exploração do todo" (FIGUEIREDO; MALTEMPI, 2019, p. 2002).

Teixeira e Becker (2001) apresentam como outra desvantagem o "[...] distanciamento entre pesquisador e dados; dados qualitativos passariam a ser analisados de forma quantitativa e, por fim, ocasionariam uma homogeneidade entre os métodos de análise de dados, inibindo a criatividade do pesquisador" (TEIXEIRA; BECKER, 2001, p. 96). Ainda nessa linha, Moreira (2007) ressalta que corremos o risco de perder uma das características da pesquisa qualitativa, que é a consciência reflexiva do pesquisador, afirmando que "[...] a produção de códigos e categorias por meio de um software pode mascarar o status interpretativo, dando-lhes um falso ar de existência concreta." (MOREIRA, 2007, p. 66).

Salientamos que, de modo geral, esses softwares apresentam várias funcionalidades como:

(a) tomada de notas de campo; (b) transcrição de notas; (c) codificação de segmentos de texto; (d) arquivo, busca e recuperação de segmentos de texto; (e) conexão de dados para formar agrupamentos ou redes de informação; ( $f$ redação de memorandos; (g) análise de conteúdo; e (h) geração de redes conceituais. (SCHLOSSER; FRASSON; CANTORANI, 2019, p. 540 - 541)

Como vimos, os CAQDAS podem ser explorados de diversas formas dentro de uma pesquisa, contudo destacamos que, para este artigo, buscamos as teses que os utilizaram na etapa da análise e não apenas para transcrição dos dados.

\section{Encaminhamentos metodológicos}

Este artigo teve como objetivo caracterizar, por meio de um mapeamento, a utilização de softwares de análise de dados qualitativos em teses voltadas à Educação Matemática, que foram desenvolvidas no contexto nacional, no período de 2010 a 2019. Segundo Fiorentini et al (2016, p. 18) o mapeamento é um "[...] processo sistemático de levantamento e descrição de informações acerca das pesquisas produzidas sobre um campo específico de estudo, abrangendo um determinado espaço (lugar) e período de tempo."

Uma característica importante do mapeamento, como procedimento metodológico, diz respeito a um olhar mais atento aos aspectos descritivos de um campo de estudo em detrimento dos resultados (FIORENTINI et al, 2016). Dessa forma, buscamos desvelar dados 
identificadores das teses, bem como aspectos relacionados à adoção de software de análise de dados qualitativos.

A opção pelas teses, como fonte de análise de dados, se dá, primeiramente, pelo interesse em acessar informações aprofundadas acerca dos aspectos metodológicos e, também, por ser o tipo de produção que os proponentes deste artigo, doutorandos, estão envolvidos, buscando reflexões para a produção de suas próprias teses.

A busca pelas teses foi realizada na Biblioteca Digital Brasileira de Teses e Dissertações (BDTD), em virtude de concentrar pesquisas realizadas em diversas instituições do país. Salientamos, nesse sentido, que a BDTD se consolida como uma das maiores iniciativas do mundo no que se refere à disseminação e visibilidade de pesquisas de pós-graduação.

A definição da expressão de busca, na base de dados, ocorreu após simulações iniciais com as expressões: "Educação Matemática" e "softwares de análise de dados qualitativos"; "softwares de análise de dados qualitativos"; "Educação Matemática" e "CAQDAS"; "CAQDAS". Essas tentativas não foram bem sucedidas, tendo em vista que restringiam significativamente a quantidade de teses, não identificando diversos trabalhos voltados à Educação Matemática que faziam uso de software de análise de dados qualitativos. Desta forma, optamos pelo uso da expressão "Educação Matemática" como buscador das teses, por ser um termo mais abrangente.

Além disso, foi aplicado um filtro referente ao período de investigação de 2010 a 2019. Essa delimitação atende a nossa intenção em compreender de que forma os softwares de análise de dados qualitativos vêm sendo explorados na atualidade. A pesquisa foi realizada em agosto de 2020 e resultou em 651 teses.

Com a finalidade de compor o corpus de análise, procedemos a uma primeira leitura das 651 teses a partir do resumo e da metodologia, das quais foram selecionadas 31, visto que apresentavam o uso do software para análise dos dados qualitativos.

Temos ciência de que a busca na BDTD pode não abarcar todas as teses direcionadas à Educação Matemática, considerando que o período foi delimitado, e a inspeção pela expressão "Educação Matemática" aconteceu apenas nos elementos pré-textuais, isto é, título, resumo e palavras-chave. Assim, trabalhos que, porventura, apresentam a expressão de busca somente nos elementos textuais não foram analisados neste artigo.

De modo a direcionar nossa investigação, realizamos um fichamento das teses selecionadas, evidenciando dados identificadores como "quando", "onde", "quem" produziu essas pesquisas e aspectos relacionados à adoção do software, tais como, natureza da pesquisa, metodologias e instrumentos de produção e análise de dados, quais foram utilizados, potencialidades e limitações dos softwares e quais as justificativas para o seu uso nos trabalhos. Esse fichamento foi executado em uma planilha eletrônica que possibilitou organizar e visualizar os dados das 31 teses em um único arquivo, a partir do qual elaboramos tabelas e gráficos para a análise e descrição dos dados.

Estruturamos a análise apresentando, primeiramente, dados identificadores das teses, posteriormente tratamos da adoção dos CAQDAS nas teses da área de Educação Matemática, explicitando aspectos da utilização dos softwares, da natureza da pesquisa, dos instrumentos de produção e coleta dos dados e das metodologias empregadas. Além disso, apresentamos reflexões a respeito das convergências e distanciamentos que marcaram a adoção dos softwares, em relação à literatura. 


\section{Resultados e discussões}

O primeiro resultado que nos fica evidenciado é que, das 651 teses, apenas 31 fizeram uso de CAQDAS, o que corresponde a aproximadamente 5\%. Esse resultado valida o que Figueiredo e Maltempi (2019) afirmam sobre o quantitativo ser insignificante na área da Educação Matemática.

Voltando nosso olhar para o corpus deste estudo, apresentamos, na Tabela 1, a distribuição das teses, conforme ano de publicação, frequência absoluta e relativa.

Tabela 1: Distribuição de teses relacionadas à Educação Matemática e teses que usaram software de análise de dados qualitativos, conforme $\mathrm{o}$ ano.

\begin{tabular}{c|c|c|c}
\hline Ano & Total de teses & $\begin{array}{c}\text { Teses que usaram } \\
\text { CAQDAS }\end{array}$ & Frequência Relativa \\
\hline 2010 & 28 & 2 & $7,14 \%$ \\
\hline 2011 & 38 & 0 & $0,00 \%$ \\
\hline 2012 & 49 & 3 & $6,12 \%$ \\
\hline 2013 & 69 & 3 & $4,35 \%$ \\
\hline 2014 & 69 & 1 & $1,45 \%$ \\
\hline 2015 & 93 & 3 & $3,23 \%$ \\
\hline 2016 & 73 & 2 & $2,74 \%$ \\
\hline 2017 & 76 & 5 & $6,58 \%$ \\
\hline 2018 & 86 & 9 & $10,47 \%$ \\
\hline 2019 & 70 & 3 & $4,29 \%$ \\
\hline Total geral & 651 & 31 & $4,76 \%$ \\
\hline
\end{tabular}

Fonte: Os autores (2020)

Em relação à produção de teses, entre os anos de 2010 a 2019, com pesquisas que contemplam a Educação Matemática, é observável (Tabela 1) um crescimento nos últimos anos, quando comparado com o ano de 2010. Acreditamos que isso possa estar relacionado ao crescimento de programas de pós-graduação em Educação Matemática ou Ensino de Matemática ocorridos em nosso país, pois conforme consta no Sistema de Informações Georreferenciadas da Capes - Geocapes, no ano de 2010, existiam 28 programas e, no ano de 2019, totalizavam 66 programas.

Mesmo com a expressiva quantidade de programas, consideramos baixo o número de teses que adotaram algum CAQDAS, visto que, em nossa pesquisa, não restringimos apenas a programas de Educação Matemática, mas em teses que apresentavam a expressão "Educação Matemática", e que poderiam pertencer a outros programas.

Com base nos dados compilados, evidenciamos, na Educação Matemática, uma oscilação na utilização destes softwares ao longo dos anos. Ao observarmos a frequência relativa (Tabela 1), podemos constatar que, somente no ano de 2018 , houve utilização mais expressiva de softwares de análise qualitativa em relação a 2010. Nos demais anos, a frequência relativa se manteve abaixo do ano de 2010. Assim, mesmo tendo um aumento expressivo de teses publicadas com relação a 2010, não evidenciamos um crescimento proporcional ao uso de CAQDAS. Note que, somente em 2018, o percentual de teses que utilizava CAQDAS superou o percentual de 2010. Chamamos a atenção, nesse ponto, para o fato de que, proporcionalmente, o uso não apresenta um crescimento uniforme, ou seja, a porcentagem de teses que fizeram uso, em relação ao número total de teses, não aumentou, por exemplo, no período de 2010 a 2015; observamos, anualmente, um aumento 
no número de teses, o que não refletiu no aumento da adoção de CAQDAS. Além disso, no ano de 2011, não identificamos nenhuma tese que fizesse uso de algum destes softwares.

As teses que fizeram uso de software de análise de dados qualitativos são provenientes, conforme Figura 1, de 9 Instituições de Ensino Superior (IES).

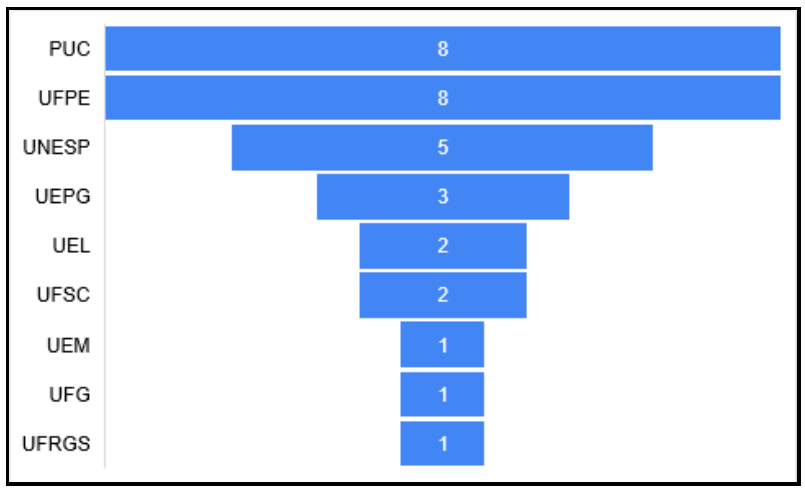

Figura 1: Instituições de Ensino Superior vinculadas às teses do corpus da pesquisa. Fonte: Os autores (2020).

Com base na Figura 1, podemos constatar que o uso de CAQDAS se concentra, especialmente, na Pontifícia Universidade Católica (PUC) e na Universidade Federal de Pernambuco (UFPE), ambas com 16 das 31 teses. A Universidade Estadual Paulista (UNESP) também apresenta um quantitativo significativo em relação ao total de teses que fez uso desse tipo de software. Já a Universidade Estadual de Ponta Grossa (UEPG), Universidade Estadual de Londrina (UEL), Universidade Federal de Santa Catarina (UFSC), Universidade Estadual de Maringá (UEM), Universidade Federal de Goiás (UFG) e a Universidade Federal do Rio Grande do Sul (UFRGS) tiveram menor representatividade.

Observando a localização das universidades de onde as teses são oriundas, evidenciamos, como se constata na Figura 2, sua distribuição em apenas 6 estados brasileiros.

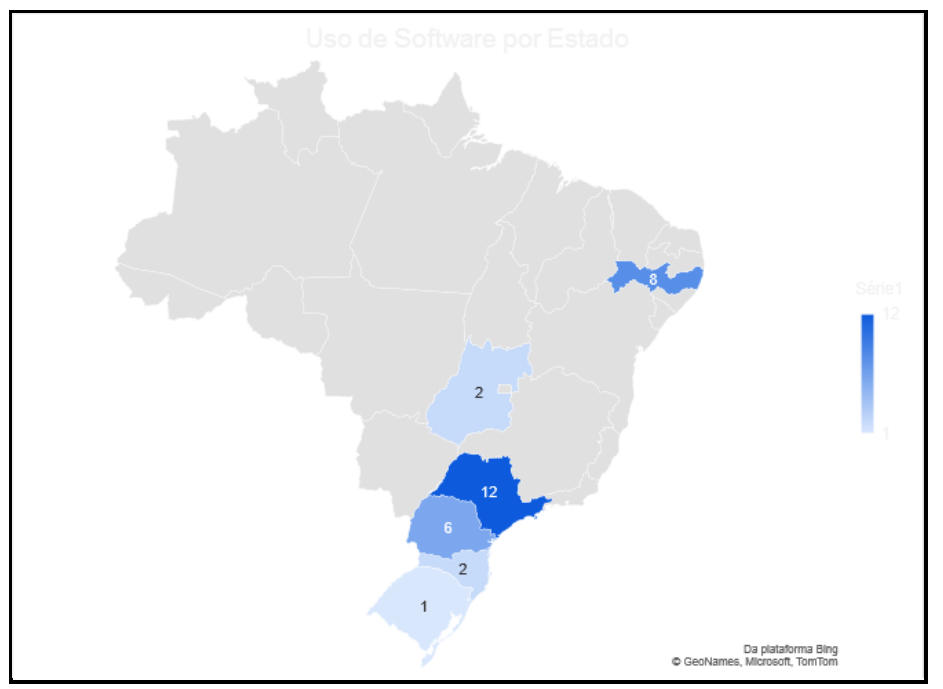

Figura 2: Distribuição geográfica das universidades que originaram as teses do corpus da pesquisa. Fonte: Os autores, 2020

Notamos que o uso de software está fortemente concentrado nas regiões Sul e Sudeste (Figura 2), com destaque para os estados de São Paulo e Paraná. Na região Nordeste temos um número expressivo, concentrado no estado de Pernambuco. Constatamos a ausência da 
utilização desse tipo de software na maioria dos estados brasileiros. Assim, inferimos que, em nosso país, não há uma prática acentuada e nem igualmente distribuída, quanto ao uso dos CAQDAS. Apresentamos, no Quadro 1, nove softwares distintos adotados pelos autores das teses.

Quadro 1: Distribuição dos softwares utilizados por autores das teses.

\begin{tabular}{|c|c|}
\hline Software & Autores (Ano da publicação da tese) \\
\hline Atlas.ti & $\begin{array}{c}\text { Faria (2012); Klüber (2012); Borssoi (2013); Silveira (2014); Ferreira (2016); } \\
\text { Kaviatkovski (2017); Silva, R. (2017); Bley (2018); Silva, K (2018); Silva, V. } \\
\text { (2018); Araújo, R. (2019); Herculano (2019) }\end{array}$ \\
\hline NVivo & $\begin{array}{r}\text { Teixeira, R. (2010); Soares (2012); Alvarenga (2013); Balvin (2015); Chiari } \\
\text { (2015); Silva, G. (2016); Araújo, A. (2017); Castro (2018) }\end{array}$ \\
\hline CHIC & $\begin{array}{r}\text { Rodrigues (2018); Roma (2010); Souza, M. (2018); Souza, R. (2018); } \\
\text { Teixeira, J. (2015) }\end{array}$ \\
\hline EVOC 2000 & Araújo, N. (2013) \\
\hline GEPHI & Gomes (2017) \\
\hline Iramuteq & Oliveira (2019) \\
\hline MAXQDA & Silva, P. (2018) \\
\hline SPSS & Lula (2017) \\
\hline WebQDA & Teixeira, L. (2018) \\
\hline
\end{tabular}

Fonte: Os autores (2020)

Ao analisar o nosso corpus, observamos que o software de análise qualitativa mais presente nas teses investigadas é o Atlas.ti, seguido por NVivo e CHIC, nesta ordem, sendo que estes três programas computacionais respondem por, aproximadamente, $80 \%$ dos trabalhos. A predominância do Atlas.ti e NVivo também foi constatada em pesquisas nas áreas de Educação e Enfermagem, conforme Paula, Viali e Guimarães (2016) e Salvador et al. (2019), respectivamente. Esse resultado mostra uma aproximação ao panorama apontado por Lage (2011) de que esses são os CAQDAS mais populares no Brasil.

Quadro 2: Distribuição das teses analisadas, conforme software e instituição proveniente

\begin{tabular}{|c|c|}
\hline Softwares & Instituição (número de teses) \\
\hline Atlas.ti & PUC (1), UEL (1), UEPG (3), UFPE (5), UFSC (2) \\
\hline NVivo & PUC (1), UFG (1), UFRGS (1), UNESP (5) \\
\hline CHIC & PUC (5) \\
\hline EVOC 2000 & UEM (1) \\
\hline GEPHI & UFPE (1) \\
\hline MAXQDA & UFPE (1) \\
\hline SPSS & PUC (1) \\
\hline WebQDA & UEM (1) \\
\hline Iramuteq & UFPE (1) \\
\hline
\end{tabular}

Fonte: Os autores (2020)

A partir do Quadro 2, podemos observar que a utilização do Atlas.ti e do NVivo está disseminada em diferentes instituições, enquanto os outros softwares tiveram seu uso em 
apenas uma instituição, destacando a utilização do programa CHIC, pela PUC, com cinco teses relacionadas. Observamos, ainda, que teses provenientes da UFPE usaram diferentes softwares (Atlas.ti, GEPHI, MAXQDA e Iramuteq), fato que também ocorreu na PUC, utilizando os softwares: Atlas.ti; CHIC e SPSS.

Na sequência, apresentamos aspectos da utilização/adoção dos softwares Atlas.ti, NVivo, CHIC e os demais com menor ocorrência nas teses. Para isso, são evidenciadas especificidades dos CAQDAS, enfatizando a natureza da pesquisa, instrumentos de produção e coleta dos dados e metodologias de análise empregadas.

\section{a) O uso do software Atlas.ti}

O Atlas.ti é um software proprietário, que se destina ao arquivamento e análise de grande volume de dados na forma de textos, gráficos, áudios e vídeos. As pesquisas, reportadas nas teses que utilizaram esse CAQDAS, são de natureza qualitativa e lançaram mão de uma combinação de alguns instrumentos para a produção dos dados: questionários, entrevistas, notas/diário de campo, observações, gravações, documentos, referências bibliográficas, que perfazem montantes significativos de dados textuais. Com relação às metodologias de análise, sobressai a análise de conteúdo, a fenomenologia e a Grounded Theory. A predominância da análise de conteúdo no uso do Atlas.ti também fica evidenciada na pesquisa realizada por Paula, Viali e Guimarães (2016), bem como o uso da Grounded Theory. Um diferencial com relação a esta pesquisa, refere-se a fenomenologia, metodologia não identificada pelas pesquisadoras.

No tocante às potencialidades apresentadas para a adoção do Atlas.ti, Klüber (2012) salienta que:

No processo habitual em que se utiliza papel e marcadores de textos o trabalho com grandes quantidades de dados é extremamente árduo, principalmente pela organização espacial para posterior análise e reorganização. Além disso, por meio do software tornou-se possível visualizar, de modo mais rápido, a totalidade das unidades de significado, ficando registrados os "passos" da redução. Desde essa perspectiva, ressalto que também foi possível refazer o processo de análise quando se percebeu alguma incoerência, o que considero outra vantagem do uso do Atlas t.i. (KLÜBER, 2012, p. 93)

Corroborando com essas afirmações, Faria (2012), Silveira (2014) e Silva, V. (2018), afirmam que este software apresenta um recurso significativo para a pesquisa qualitativa, enfatizando sua importância para o manuseio, sistematização, organização e apresentação dos dados. Bley (2018) traz apontamentos no que diz respeito ao potencial do Atlas.ti na criação de categorias, e Silva, R. (2017) complementa que esse potencial permite, até mesmo, questionar os dados, a fim de responder às perguntas de investigação.

Na visão de Kavialkosvski (2017), o Atlas.ti facilita a identificação de similaridades permitindo o agrupamento por componentes. Herculano (2019, p. 123 - 124) acentua a possibilidade de "[...] organização de um volume significativo de dados, inclusive obtidos/construídos a partir de diferentes fontes". Ferreira (2016) ainda acrescenta que esse software pode ser utilizado para arquivar comentários e auxiliar o pesquisador a fazer associações com os dados. 
Na mesma perspectiva, Borssoi (2013) aponta que o uso do Atlas.ti, além de facilitar a análise dos dados, permite equilíbrio entre sensibilidade e a objetividade. Nesse sentido, Herculano (2019) e Faria (2012), afirmam em suas teses, que o Atlas.ti não realiza análise ou inferências, ficando a cargo do pesquisador a organização dos documentos bem como interpretações dos dados. Esta assertiva é corroborada por Ferreira (2016) ao afirmar que o trabalho criativo e sistemático é realizado pelo investigador.

\section{b) O uso do software NVivo}

O segundo software mais utilizado é o NVivo, que também é proprietário e permite trabalhar em vários idiomas, fazendo uso de grandes bancos de dados, a partir de arquivos em Word, PDFs, vídeos, fotos e arquivos de áudio. As teses investigadas, que usaram este CAQDAS, são de natureza qualitativa, e fazem uso de uma combinação de diferentes instrumentos de produção de dados, como: questionários, entrevistas, notas/diário de campo, observações, documentos, referências bibliográficas, que perfazem montantes significativos de dados textuais, gravações de aula e de discussões. Além disso, estão associadas, principalmente, às metodologias de análise de dados da Grounded Theory, análise de conteúdo e triangulação de dados.

Em se tratando deste software, Chiari (2015), Silva, G. (2016), Araujo (2017) e Castro (2018) relatam que sua utilização contribuiu significativamente para a organização dos dados da pesquisa e na visão de Silva, G. (2016) facilita o movimento de ir e vir constante durante a análise. Para Chiari (2015, p. 107), o NVivo "[...] permite que o pesquisador identifique conexões sutis que seriam mais difíceis sem as ferramentas automáticas do software".

Soares (2012) e Castro (2018) frisam que a utilização do software agiliza o processo de análise, possibilitando, na opinião de Araújo (2017) e Teixeira (2010), o investimento de tempo e esforço no que se faz essencial no processo de pesquisa, o trabalho intelectual, deixando o trabalho braçal a cargo do software. Sobre as contribuições elencadas, o uso do NVivo destaca-se como uma ferramenta de apoio para a pesquisa qualitativa, na qual o pesquisador não perde seu papel de análise, visto que o processo de interpretar continua sob sua responsabilidade (ARAÚJO, A., 2017; CASTRO, 2018).

\section{c) O uso do software $\mathrm{CHIC}$}

O terceiro software mais utilizado foi o CHIC, cujo nome é um acrônimo da expressão Classificação Hierárquica, Implicativa e Coesitiva, e pode ser obtido gratuitamente. O CHIC foi desenvolvido por um grupo de pesquisadores, liderados por Régis Gras, em 1992, no Instituto de Recherche des Mathématiques de Rennes (IRMAR) da Universidade de Rennes, na França.

Dentre as cinco teses que utilizaram o software $\mathrm{CHIC}$, duas delas se autodenominavam pesquisas quali-quantitativas - Roma (2010) e Teixeira (2015) - as quais afirmam ter maior ênfase na pesquisa qualitativa. Das três restantes, Souza, M. (2018), Souza, R. (2018) manifestaram ser de abordagem qualitativa, e Rodrigues (2018) não explicitou o método, ficando implícito esse enfoque. Além disso, os autores das teses utilizaram como instrumentos de obtenção de dados: questionários, entrevistas e documentos. Ressaltamos que os autores das teses que usaram o CHIC fizeram a análise dos dados recorrendo às 
seguintes metodologias: análise de conteúdo; análise implicativa; análise coesitiva; e análise documental.

Evidenciamos, nas teses analisadas, um pesquisador referência quanto à disseminação e orientação da utilização do CHIC no Brasil, o professor e pesquisador Saddo Almouloud. De acordo com esse autor, as funções essenciais do software CHIC são "extrair um conjunto de dados, cruzando sujeitos e variáveis (ou atributos), regras de associação entre variáveis, fornece um índice de qualidade de associação e de representar uma estruturação por meio de regras" (ALMOULOUD, 2015, p. 76).

Essas funcionalidades são elencadas nas teses de Roma (2010), Souza, R. (2018), Rodrigues (2018), Souza, M. (2018). Além disso, Roma (2010) frisa que o software CHIC fornece o nível de similaridade entre variáveis/atributos, o que permite analisar a existência de causalidade entre estes, observadas nos dados. Nesse sentido, Teixeira (2015) e Souza, M. (2018) salientam que o uso do software CHIC favorece a tomada de decisões, apoiadas em certa estabilidade e pertinência de respostas.

\section{d) O uso dos softwares: Gephi, SPSS, Iramuteq, EVOC 2000, WebQDA e MAXQDA}

O software Gephi, gratuito e de código aberto, possibilita a visualização e manipulação de redes e grafos. Utilizado apenas em uma tese, o seu uso foi associado ao scriptlattes, que, a partir dos dados do Currículo Lattes, gera grafos em arquivo que são processados pelo Gephi. Gomes (2017) realizou triangulação na coleta e análise dos dados em sua tese além da classificou como de abordagem mista. A natureza quantitativa, segundo o autor (GOMES, 2017, p. 66), deriva da "[...] utilização de notações matemáticas para calcular posições e relações entre os autores, uma vez que utiliza a Teoria dos Grafos para visualização da rede, e utiliza métricas de Bibliometria para análise da produção científica"; e a qualitativa, da análise de "[...] documentos e conteúdos de entrevistas com os docentes" (p. 66).

O SPSS - Statistical Package for the Social Sciences é um software proprietário de análise estatística, que disponibiliza uma biblioteca para análise de dados qualitativos. Esse CAQDAS foi utilizado por Lula (2017) em uma pesquisa de abordagem qualitativa, desenvolvida por meio de questionários e entrevistas semiestruturadas para a coleta de dados. Ele aponta que "o objetivo ao utilizar este software é construir uma tabela em que se possa averiguar toda a análise descritiva dos dados coletados [...]" (p. 121).

O Iramuteq é um software gratuito, de código aberto, que usa a interface gráfica do software $\mathrm{R}$, permite análise de dados textuais, como: análises de conteúdo, lexicometria e análise do discurso. Sua utilização, por Oliveira (2019), ocorreu em uma pesquisa qualitativa de base teórico-metodológica e sócio-histórica, tendo o questionário de associação livre de palavras como instrumento de coleta dos dados. O programa auxiliou no tratamento dos dados por meio da análise lexicográfica, análise clássica e de similitude, bem como, na elaboração de uma nuvem de palavras.

Utilizado por Araújo (2013), o EVOC 2000, sigla para Ensemble de Programmes Permettant l'Analyse de Évocations, é um conjunto de programas que permitiu à autora processar e analisar dados que foram coletados por meio de técnica de evocação livre de palavras e de questionário com perguntas abertas, em uma perspectiva qualitativa.

O WebQDA é um software computacional proprietário, que pode ser usado on-line e de forma colaborativa. Teixeira (2018) salienta que a estrutura desse CAQDAS se assemelha 
a dos programas Atlas.ti, NVivo e MaxQDA. Nessa tese, de natureza qualitativa, foram utilizados, para a produção dos dados resultante de anotações em caderno de campo, gravação em áudio e vídeo além de entrevistas, que foram analisadas mediante análise textual discursiva. A autora considera que o WebQDA auxiliou na organização e categorização dos dados, contribuindo para a análise e interpretação destes. No entanto, ela frisa que o software não pode substituir o pesquisador, sobretudo, referente à subjetividade, à criatividade e à criticidade na análise dos dados.

O MAXQDA é um software proprietário e foi utilizado por Silva (2018), em sua tese. O autor destaca que o software auxilia na organização de categorias a partir de diferentes tipos de documentos, além disso, possibilita "[...] cruzamento de dados entre sujeitos, captura de imagens específicas nos vídeos gravados, assim como na transcrição de trechos de áudios das entrevistas realizadas" (SILVA, P., 2018, p. 108). Nessa tese, o autor, por meio de uma pesquisa qualitativa exploratória, utilizou-se de vídeos, áudios e entrevistas para a coleta de dados, a partir de uma análise interacional.

\section{Refletindo sobre a utilização dos softwares}

Uma vez explicitadas as descrições da utilização dos CAQDAS nas teses em investigação, constatam-se alguns pontos de convergência e alguns distanciamentos descritos pelos autores das teses e pela literatura.

Bicudo (2012) afirma que as pesquisas voltadas à Educação Matemática apresentam, na sua maioria, uma abordagem qualitativa, o que também pôde ser observado nas teses investigadas. Além disso, o conjunto de dados apresenta três pesquisas quali-quantitativas, relacionadas aos usos dos softwares CHIC e Gephi. No tocante à análise dos dados, observamos que a análise de conteúdo foi utilizada em diferentes softwares, como Atlas.ti, NVivo, CHIC e Iramuteq. Além disso, a metodologia Grounded Theory foi utilizada somente associada aos softwares Atlas.ti e NVivo.

Um ponto relevante observado na descrição da utilização dos softwares refere-se ao potencial de exploração da diversidade de dados, percebido a partir do uso de diferentes instrumentos de coleta de dados, como: questionários, entrevistas, documentos, referências bibliográficas, gravações de áudio e vídeo, observações e notas/diário de campo. Em especial, observando os três softwares mais utilizados, evidenciamos a convergência do uso de questionários, entrevistas e documentos. Outro aspecto refere-se ao potencial relativo à exploração do volume de dados por meio dos CAQDAS, como visto nas teses de Herculano (2019), Teixeira (2015) e Kaviatkovski (2017). Esses aspectos corroboram com a literatura, como exposto por Justo e Camargo (2014), acerca deste potencial relativo à diversidade dos dados qualitativos, que incluem qualquer forma de comunicação humana escrita, auditiva ou visual, gerando um grande volume de dados a serem analisados.

No que se refere ao uso dos CAQDAS observamos, citados com mais frequência, aspectos referentes à categorização, organização, visualização, manuseio dos dados, otimização de tempo e facilitação da análise, nessa ordem. Essa constatação se assemelha ao exposto por Teixeira e Becker (2001), de que o software faria as tarefas mais mecânicas viabilizando economia de tempo, com isso permite ao pesquisador maior dedicação à exploração e à investigação dos dados. Além disso, Figueiredo e Maltempi (2019) apontam que a tecnologia possibilita melhoria quanto ao armazenamento, representação e integração, ou seja, novas perspectivas de visualização, análise e obtenção de dados. 
Percebemos uma convergência entre o uso do NVivo e Atlas.ti, em que os esforços são direcionados para a organização dos dados e criação de categorias. Já o uso do CHIC se embasa na busca por uma certa estabilidade e pertinência de resposta com base em uma análise estatística, promovendo uma similaridade entre as variáveis envolvidas, o que, segundo Almouloud (2015), pode fortalecer as pesquisas voltadas para a Educação Matemática.

A literatura apresenta inquietações no que se refere à adoção dos CAQDAS, quanto à subjetividade do pesquisador, relacionadas ao risco de se perder a consciência reflexiva (TEIXEIRA; BECKER, 2001; MOREIRA, 2007), no entanto, nenhuma das teses apontou esta fragilidade. Pelo contrário, ficou patente nas teses que a análise, o processo reflexivo e a tomada de decisão são de responsabilidade do pesquisador e o software não influencia negativamente nesse sentido, como apontado, por exemplo, por Faria (2012), Borssoi (2013), Ferreira (2016) e Teixeira (2018).

Não encontramos menção dos autores sobre dificuldades na utilização dos softwares, o que pode indicar que: a) de fato não enfrentaram dificuldades relevantes a ponto de serem reportadas; ou b) elas não foram documentadas, o que também pode indicar carência na descrição do processo de pesquisa. Esse aspecto é corroborado pelas autoras Boaler, Ball e Huama (2003) ao afirmarem que, em geral, as pesquisas não deixam claro o modo de fazer, o processo da realização, ou o que levou à escolha de determinados passos na realização de uma pesquisa.

\section{Considerações finais}

No sentido de fundamentar escolhas baseadas no panorama da adoção de softwares de análise de dados qualitativos, caracterizamos, por meio de um mapeamento, a utilização dos CAQDAS em teses voltadas à Educação Matemática, provenientes da BDTD, publicadas no período de 2010 a 2019.

No corpus investigado, evidenciamos a pouca adoção destes softwares, oscilando ao longo do período, com destaque para o ano de 2018, que apresentou utilização mais expressiva. O uso dos CAQDAS, na Educação Matemática, está principalmente concentrado nas regiões Sul e Sudeste do país, nos estados de São Paulo e Paraná, com a maior parte dos trabalhos provenientes da PUC. Observa-se ainda um número expressivo de teses vinculadas à UFPE, instituição do estado de Pernambuco.

Nas teses analisadas, identificamos o uso de nove softwares de análise de dados qualitativos distintos, com prevalência do Atlas.ti, NVivo e CHIC. Constatamos que, em várias teses, não há justificativa explícita para a adoção do CAQDAS em suas pesquisas. Entretanto, após analisá-las, é possível notar que as palavras que pulsam atreladas à utilização dos softwares foram: categorização, organização, visualização, manuseio dos dados, otimização de tempo e facilitação da análise.

Logo, nesse cenário, este artigo pode contribuir com pesquisadores das mais diversas áreas, em especial da Educação Matemática, para que venham a realizar escolhas conscientes que facilitem a construção da sua pesquisa, principalmente aquelas que utilizam grande volume e diversidade de dados. Ficou evidenciado que os softwares utilizados apresentam potencialidades específicas e se adequam ao contexto metodológico das pesquisas. Mesmo os softwares menos utilizados foram estratégicos para a pesquisa em questão, não desmerecendo seus valores enquanto ferramenta de análise de dados. 
Salientamos que a opção da adoção dos CAQDAS nas pesquisas deve levar em consideração outros elementos, além dos apresentados neste estudo, como tempo gasto com o estudo do software, valor a ser pago pela licença (caso não seja gratuito), adaptação à metodologia da pesquisa, bem como uma análise aprofundada do que o próprio software tem a oferecer. Essas potencialidades podem ser verificadas por pessoas interessadas em fazer uso, visto que a maioria dos softwares possui uma versão de demonstração, possibilitando, assim, a interatividade com o aplicativo e subsídios para a decisão de compra.

Por fim, conforme constatado nesta pesquisa, a utilização de softwares para a análise de dados qualitativos, em teses direcionadas à Educação Matemática, é pouco expressiva. Assim, destacamos a importância de pesquisas futuras que busquem avaliar o porquê da não adoção na maioria das pesquisas de doutoramento e/ou em programas de pósgraduação, bem como a ampliação do olhar para outros bancos de dados.

\section{Referências}

ALMOULOUD, S. A. O que está detrás de CHIC. In: Armando Valente e Maria Elizabeth Bianconcini de Almeida. (Org.). Uso de CHIC na formação de Educadores: à guisa da apresentação dos fundamentos e das pesquisas e foco. 1ed. São Paulo: Letra Capital, 2015, v. 1, p. 43-62.

ARAÚJO, J. L.; BORBA, M. C. Construindo Pesquisas Coletivamente em Educação Matemática. In: BORBA, M. C.; ARAÚJO, J. L. (Org.) Pesquisa Qualitativa em Educação Matemática, Belo Horizonte: Autêntica, 2004.

BICUDO, M. A. V. A pesquisa em educação matemática: a prevalência da abordagem qualitativa. Revista Brasileira de Ensino de Ciência e Tecnologia, v. 5, n. 2, 2012.

BOALER, J., BALL, D. L., \& EVEN, R. Preparing mathematics education researchers for disciplined inquiry: Learning from, in, and for practice. In A. Bishop \& J. Kilpatrick (Eds.), International handbook of mathematics education. Dordrecht, Netherlands: Kluwer. p. $491-$ 521, 2003.

FIGUEIREDO, O. A.; MALTEMPI, M. V. A integração de tecnologias digitais na pesquisa qualitativa: possibilidades e reflexões. In: BICUDO, M. A. V.; COSTA, A.P. (org). Leituras em pesquisa qualitativa. Editora Livraria da USP: São Paulo, 2019.

FIORENTINI, D.; LORENZATO, S. Investigação em Educação Matemática. Campinas: Autores Associados, 2006.

FIORENTINI, D. et al. O professor que ensina matemática como campo de estudo: concepção do projeto de pesquisa. In: FIORENTINI, D; PASSOS, C. L. B.; LIMA, R. C. R., (orgs.).

Mapeamento da pesquisa acadêmica brasileira sobre o professor que ensina matemática: período 2001-2012. Campinas, SP: FE/UNICAMP, 2016. p. 17-42.

FLICK, U. Introdução à pesquisa qualitativa. Porto Alegre: Artmed, 2009.

GARNICA, A. V. M. História Oral e Educação Matemática. In: BORBA, M. C.; ARAúJO, J. L. (Org.) Pesquisa Qualitativa em Educação Matemática. Belo Horizonte: Autêntica, 2004. 
JUSTO, A. M.; CAMARGO, B. V. Estudos qualitativos e o uso de softwares para análises lexicais. Caderno de artigos: X SIAT \& II Serpro. p. 37-54, 2014.

LAGE, M. C. Utilização do software NVivo em pesquisa qualitativa: uma experiência em EaD. ETD - Educação Temática Digital, v. 12, p. 198 - 226, 14 dez. 2011.

MOREIRA, D. A. O uso de programas de computador na análise qualitativa: oportunidades, vantagens e desvantagens. Revista de Negócios, Blumenau, v. 12, n. 2, p. 56 - 68, abril/junho, 2007.

PAULA, M. C.; VIALI, L.; GUIMARÃES, G. T. D. A pesquisa qualitativa e o uso de CAQDAS na análise textual: levantamento de uma década. Internet Latent Corpus Journal. v. 6. n. 2, p. 65 - 78, 2016.

SALVADOR, P. T. C. O. et al. Softwares de análise de dados Qualitativos utilizados nas Pesquisas da enfermagem. Texto \& Contexto Enfermagem, v. 28, 2019.

SCHLOSSER, D. F.; FRASSON, A. C.; CANTORANI, J. R. H. Software livre para análise de dados qualitativos. Revista Brasileira de Ensino, Ciências e Tecnologia, Ponta Grossa, v. 12, n. 1, p. 539 - 550, jan/abr 2019.

TEIXEIRA, A. N.; BECKER, F. Novas possibilidades da pesquisa qualitativa via sistemas CAQDAS. Sociologias, Porto Alegre, ano 3, n. 5, p. 94 - 113, jan/jun 2001.

\section{Referências do corpus}

ALVARENGA, K. B. O que dizem as pesquisas sobre o ensino e a aprendizagem de inequações. 2013. 275 f. Tese (Doutorado em Educação) - Pontifícia Universidade Católica de São Paulo, São Paulo, 2013.

ARAÚJO, A. R. Educação Matemática a Distância: A reconstrução de conceitos e práticas pedagógicas no ensino do número. 2017. 170 f. Tese (Doutorado em Educação) Universidade Federal do Rio Grande do Sul, Porto Alegre, 2017.

ARAÚJO, N. S. R. Representações sociais de professores de matemática e alunos da educação de jovens e adultos sobre esta modalidade de ensino e a matemática. 2013. 139 f. Tese (Doutorado em Educação para a Ciência e a Matemática) - Universidade Estadual de Maringá, Maringá, 2013.

ARAÚJO, R. K. S. Avaliação da aprendizagem na educação online: construindo elementos para um avaliar interativo-mediador. 2019. 468 f. Tese (Doutorado em Educação Matemática e Tecnológica) - Centro De Educação, Universidade Federal de Pernambuco, Recife, 2019.

BALVIN, F. A. P. Práticas algébricas no contexto de modelagem compreendida como proposta pedagógica. 2015. 217 f. Tese (Doutorado em Educação Matemática). Universidade Estadual Paulista Júlio de Mesquita Filho, Rio Claro, 2015.

BLEY, D. H. P. Panorama dos elementos influenciadores do processo da adoção de tecnologias por professores. 2018. 262 f. Tese (Doutorado em Educação Matemática e Tecnológica) - Universidade Federal de Pernambuco, Recife, 2018.

BORSSOI, A. H. Modelagem Matemática, Aprendizagem Significativa e Tecnologias: articulações em diferentes Contextos Educacionais. 2013. 256 f. Tese (Doutorado em Ensino de Ciências e Educação Matemática) - Universidade Estadual de Londrina, Londrina, 2013. 
CASTRO, A. L. Matemática e o currículo da era digital: os desafios para a inovação na prática educativa. 2018. 291 f. Tese (Doutorado em Educação para a Ciência) - Universidade Estadual Júlio de Mesquita Filho, Bauru, São Paulo, 2018.

CHIARI, A. S. S. O papel das tecnologias digitais em disciplinas de álgebra linear a distância: possibilidades, limites e desafios. 2015. 206 f. Tese (Doutorado em Educação Matemática) Universidade Estadual Paulista Júlio de Mesquita Filho, Rio Claro, 2015.

FARIA, E. C. Do ensino presencial ao ensino a distância: a inovação na prática pedagógica de professores de Matemática. 2012. 154 f. Tese (Doutorado em Educação Matemática) Faculdade de Ciências Exatas e Tecnologia, Pontifícia Universidade Católica de São Paulo, São Paulo, 2012.

FERREIRA, C. R. A Modelagem Matemática na Educação Matemática como eixo Metodológico da Prática do professor de Matemática. Tese (Educação) - Universidade Estadual de Ponta Grossa, Ponta Grossa, 2016.

GOMES, C. H. P. Redes Sociais de Coautoria Científica: reflexões sobre inovação na docência da Educação Superior. 2017. 206 f. Tese (Doutorado em Educação Matemática e Tecnológica) - Universidade Federal de Pernambuco, Recife, 2017.

HERCULANO, E. V. S. Coreografias didáticas da formação continuada de professores dos anos iniciais do ensino fundamental para o uso pedagógico das tecnologias digitais: elementos para uma prática formativa inovadora. 2019. 206 f. Tese (Doutorado em Educação Matemática e Tecnológica) - Universidade Federal de Pernambuco, Recife, 2019.

KAVIATKOSVSKI, M. A. C. As práticas de modelagem matemática no âmbito do ensino fundamental: um olhar a partir de relatos de experiência. 2017. 164 f. Tese (Doutorado em Educação) - Universidade Estadual de Ponta Grossa, Ponta Grossa, 2017.

KLÜBER, T. E. Uma metacompreensão da modelagem matemática na educação matemática. 2012. Tese (Doutorado em Educação Científica e Tecnológica) - Universidade Federal de Santa Catarina, Florianópolis, 2012.

LULA, K. P. A formação dos formadores de professores de matemática: um estudo na licenciatura em matemática do IFG - Campus Goiânia. 2017. 371 f. Tese (Doutorado em Educação) - Pontifícia Universidade Católica de Goiás, Goiânia, 2017.

OLIVEIRA, V. C. Sentidos de docência universitária nas licenciaturas presenciais e a distância: relações com a identidade e a profissionalidade docente. 2019. 243 f. Tese (Doutorado em Educação Matemática e Tecnológica) - Universidade Federal de Pernambuco, Recife, 2019.

RODRIGUES, M. R. Estudo sobre as concepções de professores do ensino básico em relação à aleatoriedade e probabilidade. 2018. 229 f. Tese (Doutorado em Educação Matemática) Pontifícia Universidade Católica de São Paulo, São Paulo, 2018.

ROMA, J. E. As representações sociais dos alunos da licenciatura em matemática sobre a profissão docente. 2010. 250 f. Tese (Doutorado em Educação) - Pontifícia Universidade Católica de São Paulo, São Paulo, 2010.

SILVA, G. H. G. Equidade no acesso e permanência no ensino superior: o papel da educação matemática frente às políticas de ações afirmativas para grupos sub-representados. 2016. 359 
f. Tese (Doutorado em Educação Matemática) - Instituto de Geociências e Ciências Exatas, Universidade Estadual Paulista Júlio de Mesquita Filho. Rio Claro, 2016.

SILVA, K. C. Avaliação das políticas públicas para educação a distância no Brasil: análise dos elementos para construção de um modelo de avaliação de efetividade da Universidade Aberta do Brasil. 2018. 279 f. Tese (Doutorado em Educação Matemática e Tecnológica) Universidade Federal de Pernambuco, Recife, 2018.

SILVA, P. A. Espaço flexível de aprendizagem no ensino superior: perspectivas docentes sobre a sala de aula. 2018. 195 f. Tese (Doutorado em Educação Matemática) - Centro de Educação, Universidade Federal de Pernambuco, Recife, 2018.

SILVA, R. J. S. Construção de indicadores para gestão de tecnologia de informação e comunicação na educação: um estudo de caso. 2017. 230f. Tese (Doutorado), Universidade Federal de Pernambuco, Recife, 2017.

SILVA, V. S. Modelagem Matemática na formação inicial de pedagogos. 2018. 189f. Tese (Doutorado em Educação) - Universidade Estadual de Ponta Grossa, Ponta Grossa, 2018.

SILVEIRA, E. A modelagem em educação matemática na perspectiva CTS. 2014. 203 f. Tese (Doutorado em Educação Científica e Tecnológica) - Centro de Ciências da Educação, Universidade Federal de Santa Catarina, Florianópolis, 2014.

SOARES, D. S. Uma abordagem pedagógica baseada na análise de modelos para alunos de biologia: qual o papel do software? 2012. 341 f. Tese (Doutorado em Educação Matemática) Instituto de Geociências, Universidade Estadual Paulista Júlio de Mesquita Filho, Rio Claro, 2012.

SOUZA, M. A. S. PIBID: significados na formação inicial de professores de matemática. 2018. 207 f. Tese (Doutorado em Educação Matemática) - Pontifícia Universidade Católica de São Paulo, São Paulo, 2018.

SOUZA, R. A. Educação Financeira: Uma abordagem centrada na modelagem matemática. 2018. 125f. Tese (Doutorado em Educação Matemática) - Pontifícia Universidade de São Paulo, São Paulo, 2018.

TEIXEIRA, J. Um estudo diagnóstico sobre a percepção da relação entre educação financeira e matemática financeira. 2015. 160f. Tese (Doutorado em Educação Matemática) - Pontifícia Universidade de São Paulo, São Paulo, 2015.

TEIXEIRA, L. A. Um estudo a respeito da aprendizagem científica em uma escola de 1 ciclo de Portugal. 2018. 122 f. Tese (Doutorado em Ensino de Ciências e Educação Matemática) Universidade Estadual de Londrina, Londrina, 2018.

TEIXEIRA, R. A. G. Matemática Inclusiva? O processo ensino aprendizagem de matemática no contexto da diversidade. 2010. 423 f. Tese (Doutorado em Ciências Humanas) - Universidade Federal de Goiás, Goiânia, 2010. 